\title{
Consistency of a Lorentz-violating extension of the photon sector
}

\section{Marco SCHRECK*}

Institute for Theoretical Physics

Karlsruhe Institute of Technology (KIT), Germany

E-mail: marco.schreck@kit.edu

In this article a Lorentz-violating extension of quantum electrodynamics (QED) will be discussed. The modification is CPT-even, power-counting renormalizable and can be embedded into the gauge group of the standard model. We review previously obtained results concerning the consistency of physically interesting sectors of this modified theory.

The conclusion is that at least two of the three presented cases are consistent, i.e. microcausal and unitary, for a particular range of deformation parameters.

The 2011 Europhysics Conference on High Energy Physics, EPS-HEP 2011,

July 21-27, 2011

Grenoble, Rhône-Alpes, France

${ }^{*}$ Speaker. 


\section{The standard model and Lorentz violation}

In light of the Europhysics Conference on High Energy Physics 2011 in Grenoble, the standard model of elementary particle physics has proven to be very successful, the only remaining missing link being the Higgs particle. Much effort is put into the search for physics beyond the standard model, such as supersymmetry. However, no conclusive evidence has been found so far. Because of this it might not be any wonder why most phenomenologists are not interested in Lorentz symmetry violation. Up to now Lorentz symmetry has been an established property of the standard model. The recent detection of superluminal muon neutrinos by the OPERA collaboration [1] is, indeed, remarkable, but the physics community remains sceptical about the result.

Lorentz invariance could be the low-energy property of a fundamental theory. String theory and loop quantum gravity are possible prototypes, but a complete theory of everything is still out of reach. Therefore, it makes sense to parameterize a violation of Lorentz symmetry in the framework of the standard model, which is established and well-known. From this approach the Lorentzviolating deformation of the standard model [2] arises.

\section{Modified quantum electrodynamics and modified Maxwell theory}

We consider a deformation of QED including a modified Maxwell term and the standard Dirac term. The modified Maxwell theory parameterizes Lorentz violation in the photon sector, whereas the fermion sector and the fermion-photon interaction are conventional. This modified QED action reads:

$$
\begin{gathered}
S_{\text {modQED }}=S_{\text {modMax }}+S_{\text {standDirac }}, \\
S_{\text {modMax }}=\int_{\mathbb{R}^{4}} \mathrm{~d}^{4} x\left(-\frac{1}{4} F^{\mu v} F_{\mu \nu}-\frac{1}{4} \kappa^{\mu v \rho \sigma} F_{\mu v} F_{\rho \sigma}\right), \\
S_{\text {standDirac }}=\int_{\mathbb{R}^{4}} \mathrm{~d}^{4} x \bar{\psi}(x)\left[\gamma^{\mu}\left(\mathrm{i} \partial_{\mu}-e A_{\mu}(x)\right)-M\right] \psi(x),
\end{gathered}
$$

with the field strength tensor $F_{\mu v}(x) \equiv \partial_{\mu} A_{v}(x)-\partial_{v} A_{\mu}(x)$, the $\mathrm{U}(1)$ gauge field $A_{\mu}(x)$, and the spinor field $\psi(x)$. All fields are defined in Minkowski space. The standard Maxwell action is deformed by the second term of Eq. (2.1b), which involves the "tensor" $\kappa^{\mu v \rho \sigma}$. Since the latter can be considered as a fixed background field penetrating the vacuum, this term obviously breaks Lorentz invariance. The consequence is that the dispersion law of the photon is modified and this may lead to a dispersive or even a birefringent vacuum.

By symmetry arguments the tensor $\kappa^{\mu v \rho \sigma}$ has 19 independent components, whose values do not follow from the theory itself, but have to be measured or constrained by experiments. Ten of these 19 components lead to a birefringent vacuum and they are bounded at the $10^{-32}$-level. Therefore, in general, all considerations concerning modified Maxwell theory are restricted to the nonbirefringent sector that is characterized by the remaining nine parameters.

\section{Theoretical consistency of the deformed quantum electrodynamics}

Although many experiments have been performed in order to measure Lorentz violation in the photon sector, the consistency of such a modified theory is a priori unclear. In Ref. [3] different cases of modified Maxwell theory were investigated and the results will be reviewed below. 


\subsection{Isotropic sector}

The isotropic theory is distinguished by the following modified dispersion relation of the photon:

$$
\omega(\mathbf{k})=\mathscr{B}|\mathbf{k}|, \quad \mathscr{B}=\sqrt{\frac{1-\widetilde{\kappa}_{\mathrm{tr}}}{1+\widetilde{\kappa}_{\mathrm{tr}}},}
$$

where $\widetilde{\kappa}_{\text {tr }}$ is the only Lorentz-violating parameter unequal to zero. The first issue to consider is microcausality, that is whether the commutator of physical electric and magnetic fields $\mathbf{E}$ and $\mathbf{B}$ vanishes outside the null-cone. Besides a certain tensor structure, such commutators involve a scalar commutator function that vanishes everywhere, except on the modified null-cone itself. This guarantees microcausality. The next aspect is unitarity, which is shown to be valid via the optical theorem - at least at tree-level. Since signals only propagate for $\widetilde{\kappa}_{\text {tr }} \in(-1,1]$, both properties hold for this parameter regime.

\subsubsection{Anisotropic parity-even sector}

An anisotropic parity-even case with one single Lorentz-violating parameter $\widetilde{\kappa}_{33}$ is characterized by the modified photon dispersion law

$$
\omega\left(k_{\perp}, k_{\|}\right)=\sqrt{k_{\perp}^{2}+\mathscr{D}^{2} k_{\|}^{2}}, \quad \mathscr{D}=\sqrt{\frac{1-2 \widetilde{\kappa}_{33} / 3}{1+2 \widetilde{\kappa}_{33} / 3}},
$$

where $k_{\|}$and $k_{\perp}$ are components of the photon three-momentum that are parallel and perpendicular to the direction of the background field. An investigation of microcausality and unitarity analogous to the isotropic case leads to the result of a consistent theory for $\widetilde{\kappa}_{33} \in(-3 / 2,3 / 2]$.

\subsubsection{Parity-odd sector}

The parity-odd case is birefringent to quadratic order in the Lorentz-violating parameter $\mathscr{E}$ :

$$
\omega_{1}\left(k_{\perp}, k_{\|}\right)=\mathscr{E} k_{\|}+\sqrt{k_{\perp}^{2}+\left(1+\mathscr{E}^{2}\right) k_{\|}^{2}}, \quad \omega_{2}\left(k_{\perp}, k_{\|}\right)=\mathscr{E} k_{\|}+\sqrt{1+\mathscr{E}^{2}}|\mathbf{k}| .
$$

Here $k_{\|}$and $k_{\perp}$ are the photon momentum components parallel and orthogonal to one of the two preferred spacetime directions that are characterized by the background field. The photon polarization vectors in this case are highly deformed. Both are interweaved with the background field and one even has a longitudinal part. This sector will be investigated in [4]. At the moment, a final and definite conclusion about its consistency is still outstanding.

\section{References}

[1] T. Adam et al. [OPERA Collaboration], "Measurement of the neutrino velocity with the OPERA detector in the CNGS beam," arXiv:1109.4897 [hep-ex].

[2] D. Colladay and V.A. Kostelecký, "Lorentz-violating extension of the standard model," Phys. Rev. D 58 (1998) 116002, arXiv:hep-ph/9809521.

[3] F. R. Klinkhamer, M. Schreck, "Consistency of isotropic modified Maxwell theory: Microcausality and unitarity," Nucl. Phys. B 848 (2011) 90, arXiv:1011.4258 [hep-th].

[4] M. Schreck, "Analysis of the consistency of parity-odd nonbirefringent modified Maxwell theory," to be published soon. 